\title{
Morse Code Detection Using Eye Blinks
}

\author{
Prof. Vijay Jumb, Charles Nalka, Hasan Hussain, Ricky Mathews \\ Dept. Computer Engineering Mumbai University,Mumbai, India
}

\begin{abstract}
A novel approach for Morse code generation with its interpretation in English language is done. The Morse code is generated with the help of eyes. Every time a person/user blinks his/her eyes an output of dash and dot is generated which is Morse code. OpenCV and Dlib libraries are used with facial landmark detection for eye blink detection. There are approximately 21 million people of India suffering from one or the other kind of disability. Within a blink these people can express themselves to the world more gracefully. This system allows user to be understand easily by anyone who understands human language well.
\end{abstract}

Index Terms—-Morse code, OpenCV, Eye Blink

\section{INTRODUCTION}

Speech disorders can affect the way a person trying to speak clearly and distinctly to form words. Speech disorders can be due to certain medical ailments starting from brain damage, stroke to paralysis, and several other diseases. It can also result in collapse of central nervous system's peripheral damage during accidents which can leave a person completely not able to communicate. In paralysis, the ability to control muscle movement is limited even around the eye muscles and blinking is the only way for patients to communicate. In such conditions, a system which is convenient as well as easy is required for the patient. Since speech impairment is not directly harmful to the immediate health of the patient, it is not taken in consideration as much it should be taken in the development of a medical device. Human communications are usually through voices and expressions. Besides natural languages (e.g., spoken languages, written languages), sign language consisting of facial expressions and body gestures is another primary means of communication for the special people below special conditions; meanwhile, the body gesture is very meaningful to improve the effectiveness of human interactions. The finger gesture in particular is a relatively stable composed style hand movements. The different technologies used for implementing the communication between paralyzed patients and the people who attend and care for them are activated by the mouth joysticks, powered breathing straws, tongue movement analysis, switch mounted near the user's head, etc. These systems are costly to implement, increase stress on the patients, and need skilled labor to set up and maintain the system for proper functioning. Hardly any devices have been developed that can address this issue in a patient-friendly and cost-effective manner. This work aims to design a simple and costeffective software for patients suffering from speech disorder as well as people with the only option of eye movements/blinks using eye blinks of the person. Eyeblink at regular intervals can be interpreted for forming words for communication. An efficient real-time blink detection algorithm can be used for almost any Purpose,such as turning on/off electrical appliances at home. The Morse code was developed in the early 19th century when the people did not have any idea of constructing circuits to send voice messages from one place to another. The telegraph systems were means of sending and receiving messages with help of electronic impulses. The Morse code was named so after its inventor, Samuel F B Morse. It continues to be the easiest, efficient, and affordable means of communication as the devices required by it was very simple. This method proved that proficiency in English was a requirement to communicate with the rest of the world. Later on, this code was accepted globally and a common International Morse code has been developed and used. Morse code was popularly known as the language of dots and dashes. Years later, this language came up with improvised versions like transmitting text information as a series of toggling tones, changing brightness levels, or ticks that can be directly decoded by a skilled listener or an observer without the use of any special type of equipment. It has been the most simplest and inexpensive method of transmitting and receiving messages. Over the years this method was mainly used in radio communication but today this methodology has many applications in aviation, navy, and assistive methodology like helping people with disabilities to 
communicate. We aim to give support to people with speech disorders but there are some military-strategic situations where it can be useful too. Here within eye-blinks, the user can easily express himself/herself. This process seems to be time-consuming but it's not, once with some practice the user will get fast at this.

\section{LITERATURE REVIEW}

There's a lot of technology used in face detection or about how to generate morse code with various gestures and all.There are a lot of projects which can detect hand gestures to detect language which in short a sign language detection and interpretation is performed. We found a few systems that generated morse code where everyone was unique and with their algorithms and technology. Those various techniques are infrared switch which was used to generate a sound from where according to sound intensities or frequencies are

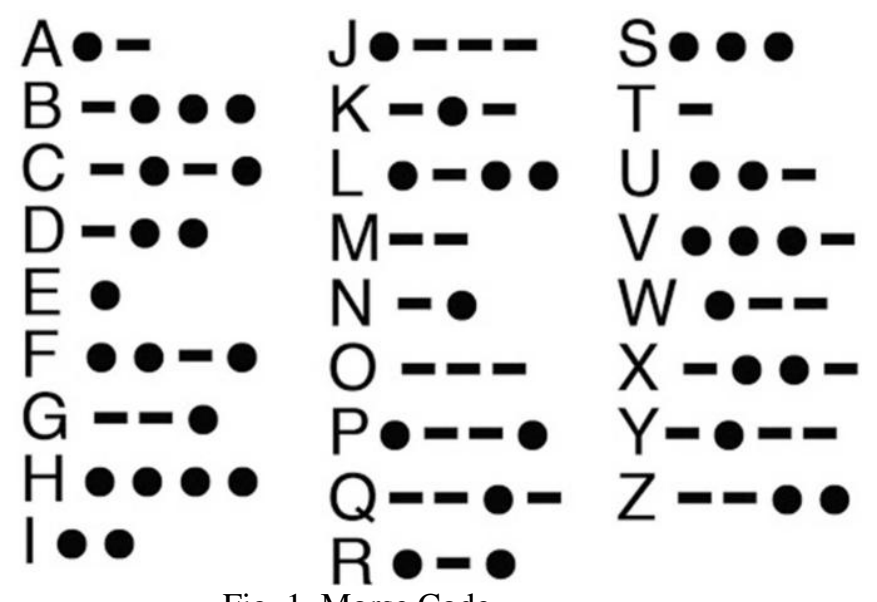

Fig. 1. Morse Code.

detected and morse code is generated according to it. The next technique is Finger gesture recognition and Mouth movements where image processing is applied to extract features carefully.Now, a brief about these systems will be done here. The generation of morse code with images used image processing techniques and fuzzy algorithms [1]. Various transformations are performed on an image, the first transformation is color space transformation that is RGB(red green blue) to HSL(hue, saturation, lightness), then the second transformations is Hue panel, the next i.e. 3rd transformations is saturation panel. This saturation panel gives the binary image of the skin area filled with the pixel value of one. The next operation is a combination of results of 3rd and 2nd operation it is the logical operation, the main aim of this logical operation is to get the skin area. From here the co-ordinates of lips are extracted and with the help of fuzzy algorithms, it is checked or verified whether the mouth is closed or open. Along with this, it is also checked that, for how much time the mouth is opened, with this it is decided that the output should be a dash or a dot which is morse code. The next system is finger gesture recognitions, a camera is employed to capture a sequence of video frames, then it will recognize the finger gestures from these frames and convert it to morse code where it will further convert the corresponding morse code to readable text [2]. Here SVM bases kernel functions and BPNN i.e. back propagation neural network has experimented. It is a 3-layer neural network that is distinguished under supervised learning. The sigmoid function is used for training. BPNN is more about error managing. In short RBF kernel and BPNN algorithms are the two classifiers used to recognize human finger gesture. When the fingertip is touched the threshold is triggered and according to the duration it is touched, the morse code is generated. These are a couple of techniques discussed above.

\section{A. Limitation in Existing Systems}

The system and technique used above are restricted to a few people such as people handicapped where they cannot use their hands or finger and cannot move their jawline due to a fracture or some of the other reasons. These kinds of things restrict the proposed system to be completely not applicable to disabled people. Another limitation or drawback of their system is that their system cannot speak, this major limitation

considering the advancement of today's technology is also a point to inspire us to make such an audible system which can speak i.e. to translate morse code to a human communicative language.

\section{B. Objective And Scope}

A person has to be steady and maintain a proper distance from where the camera distance will be able to detect the person's face and start capturing and detecting him/her. If this system would not exist then the person was in a very difficult situation to communicate with the other person. A disabled person generally uses sign language, here the problem is that not everyone is aware and known of sign language so it becomes difficult for every person to communicate. With this software, a person can express themselves faster and easier. It is important to solve the problem for such people who can communicate with every person in the world. As we saw in the current systems they are not easy with people who have spinal cord injuries and all. Also, the efficiencies are not up to the mark for their current systems available. With help of SVMs, we can have 
very high efficiency. This software is also inexpensive and can be deployed on any Linux, ios, and windows system easily. These all benefits like cost-effectiveness and easy deployment of the system are the main advantage of the system. Another requirement which is most important i.e. inbuilt camera is required in the system. Nowadays every other laptop has a camera, if not available then USB extended cameras are also available. This component of the system is most costly whereas others are inexpensive. This system will be very efficient for the people who can easily blink their eyes and have some of the other physical disability. This system can be made more accurate with SVM i.e. support vector machines, etc. we can use NLP natural processing language for better impact. Due to the technological advancement, its scope is infinite we can completely make it voice activated and navigated.

\section{PROPOSED DESIGN}

\section{A. Analysis}

Our project is based on detection of morse code from eye blinks. As discussed in the previous section there are many ways to detect a morse code like by using mouth, fingers or by using hands. We will be detecting morse code from a pattern of eye blink. Before detecting morse code from eye blinks, we first have to detect eyes. Morse code detection has 3 major steps:

1) Locating eyes

2) determining Eye Aspect Ratio

3) detecting morse code from blinks

\section{B. Methodology}

1) Locating Eyes: So, to locate an eye we first have to detect face, for face detection we will use a pre trained dataset "facial landmarks" [3] [8]. For each frame from the input video stream we have to resize and convert the frames into gray scale image, from these frames using dlib library we will be detecting the facial structures and locate the position of the eye. Facial landmarks refers to 68 (x, y) coordinates of the different facial features like left eyebrow, right eyebrow, left eye, right eye, nose, mouth and jaw line as we can see in the figure 2

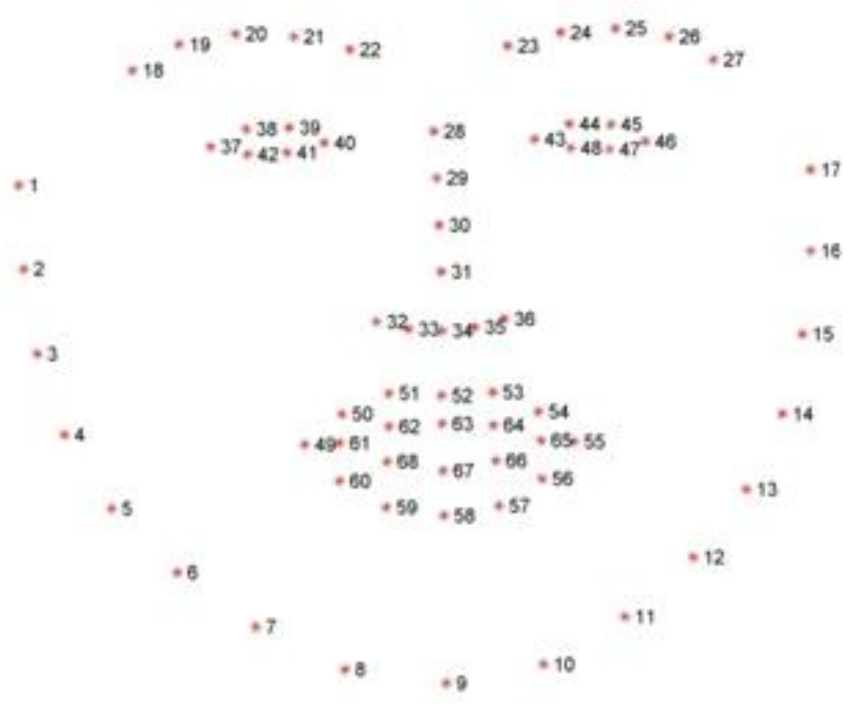

Fig. 2. Facial Landmarks.

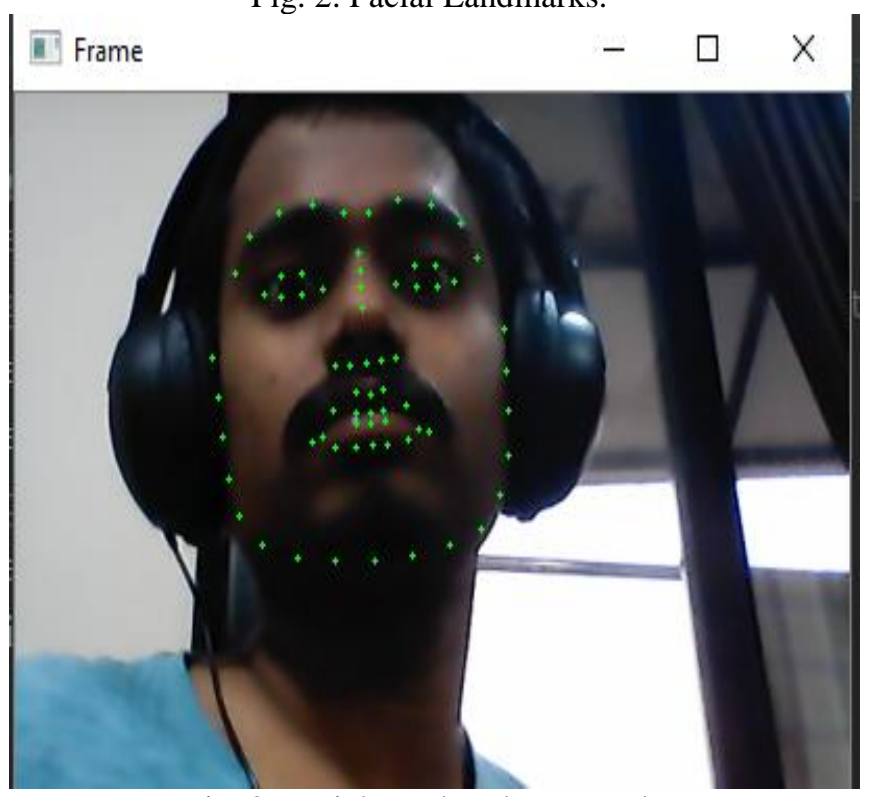

Fig. 3. Facial Landmarks Example.

Now as each facial structure are represented by points, we will apply a computer vision technique that is image extraction and extract those points which comes under eyes, as from figure $*$ we can see that points $37-42$ are representing left eye and points $43-48$ are representing right eye, we will be considering only these points.

2) Determining Eye Aspect Ratio: To determine when an eye is closed or when an eye is opened, we will be computing a metric called Eye Aspect Ratio. EAR is 
computed to calculate the horizontal and vertical distance of an eye. Each eye consists of $6(x, y)$ point as can be seen in the figure 4.

The two pair of vertical point is for computing vertical distance and single pair of points for horizontal distance, A formula can be derived based on this points called Eye aspect

ratio [4].

$$
E A R=\frac{|p 2-p 6|+|p 3-p 4|}{2|p 1-p 4|}
$$

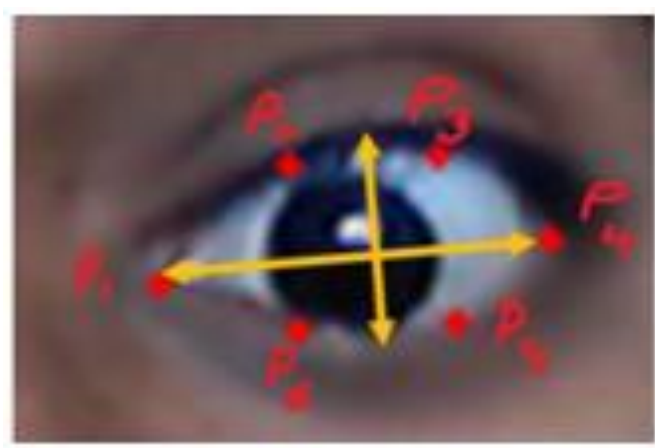

Fig. 4. Single Eye Representation.

In the above equation point's p1 - p6 are 2D facial landmarks, where the numerator is for vertical distance and the denominator is for horizontal distance. Generally when the eye remains opened as in figure 5 the EAR value remains constant and when the eye is closed as in the figure 6 the EAR value approaches to zero. Using this simple computation we can determine whether a blink has occurred or not.

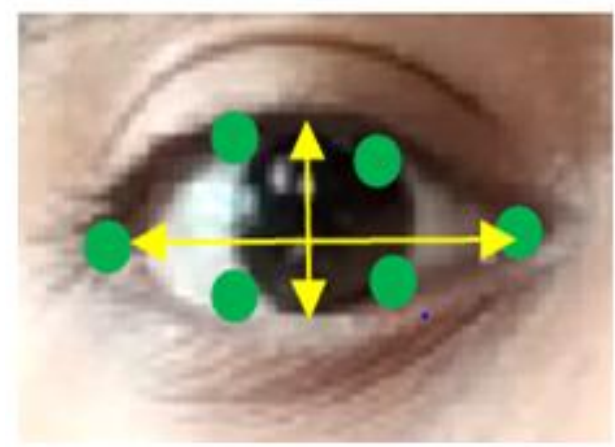

Fig. 5. Opened Eye Landmark.

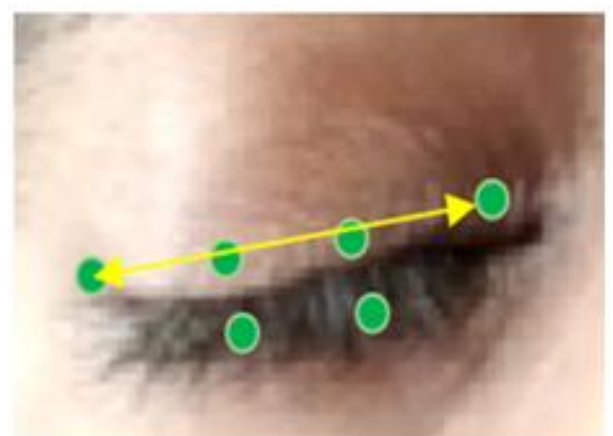

Fig. 6. Closed Eye Landmark.

In the below graph (figure 7) as we can see, the EAR remains constant untill the eye is opened as soon as the eye is closed the EAR decreases and dramatically reaches to 0 . Consider a threshold frequency as 0.2 in the below graph, as soon as the value of EAR goes below the threshold value i.e. 0.2 (when the eye is closed) and approaches to 0 and then increases, going above the threshold value (when the eye is opened) we can say a blink has occurred. Using this simple computation of decreasing and increasing value of the EAR value we can determine whether a blink has occurred or not.

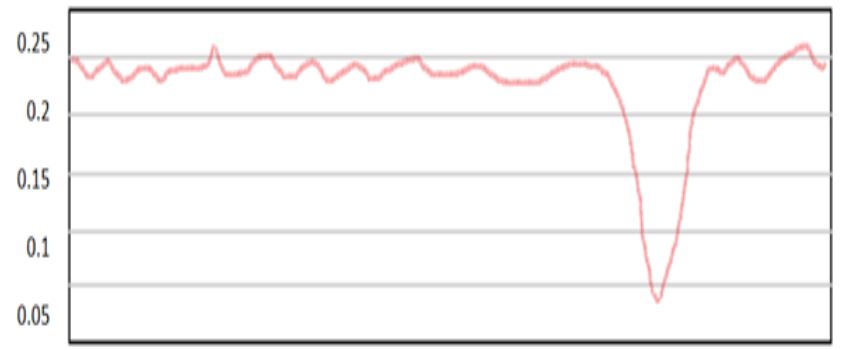

Fig. 7. EAR Threshold Graph.

3) Detecting Morse Code From Blinks: After detection of blink is implemented using EAR, now we will understand how the system will generate morse code from these patterns of blinks. So when the user executes the code, input will be taken from the webcam or a recorded video, from this, eyes are detected using facial landmarks. Then analysis of data and computation of EAR values is performed. Morse code is generated from the pattern of blinks that is when the user closes his eyes for 1 second a '.'(dot) is detected and when the user closes his eyes for 2 seconds a '-'(dash) is detected. This dot and dash are stored and then converted into English language. 


\section{Flow Chart}

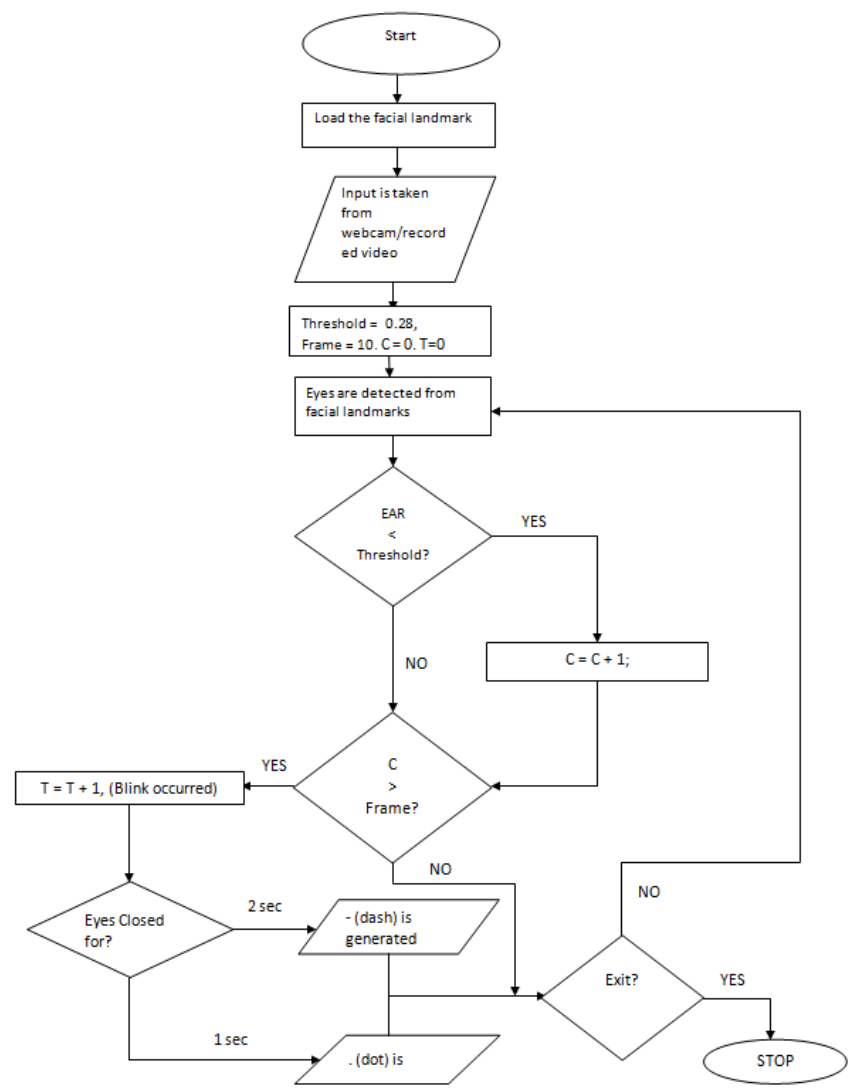

Fig. 8. Flow Chart.

\section{CONCLUSION}

We have seen that Eye Aspect ratio has proven to be very effecting in detecting blinks, other than the traditional method to detect blinks. Morse code blink detection can be very useful for disabled people, paralysed people with some eye movement left. With the help of better algorithms and techniques, we discussed, blinks can be detected more accurately. Further these blinks are being converted to morse code. For patients with lower communication requirements, a special code could be easily devised where specific pattern would represent a special phrase or commands. There is an array of basic life functions can easily be communicated by a couple of commands which may be effortlessly learned by the patient. This system can be implemented not only to people who have body disorders as well as speaking disorders but can also be implemented in places like libraries, hospitals, or schools. This system may be useful for spies or people working in military to convey a secret message without enemy's knowledge.

\section{REFERENCES}

[1] Image Morse Code Text Input System , Shih-Chung Chen, Chung-Min Wu, Shih-Bin Su, Department of Electrical Engineering, Southern Taiwan University Department of Electronic Engineering, Kun Shan University 1 No.1, Nantai St, Yung-Kang Dist., Tainan, 710, Taiwan R.O.C. 2 No.949, Dawan Rd., Yongkang Dist., Tainan, 710, Taiwan R.O.C.

[2] Morse Codes Enter Using Finger Gesture Recognition Ricky Li, Minh Nguyen, Wei Qi Yan Department of Computer Science Auckland University of Technology, Auckland, 1010 New Zealand.

[3] One millisecond face alignment with an ensemble of regression trees V. Kazemi, J. Sullivan Published 2014 Computer Science 2014 IEEE Conference on Computer Vision and Pattern Recognition

[4] Adrian Rosebrock. Eye blink detection with opencv, python, and dlib. URL https:// www.pyimagesearch.com /2017/04/24/eye-blink-detectionopencv- python-dlib/.

[5] Alternative Voice Communication Device using Eye Blink Detection for People with Speech Disorders Srividhya G, Murali S, A. Keerthana, Jaya Rubi

[6] A Novel Method for Eye Tracking and Blink Detection in video frames Leo Pauly, Deepa Sankar Division of Electronics and Communication Engineering School of Engineering Cochin University of Science and Technology

[7] Eye Blink Detection Using Local Binary Patterns Krystyna Malik, Bogdan Smolka Silesian University of Technology, Department of Automatic Control Akademicka 16 Str, 44-100 Gliwice, Poland

[8] Real-Time Eye Blink Detection using Facial Landmarks Tereza Soukupova and Jan ' Cech • Center for Machine Perception, Department of Cybernetics Faculty of Electrical Engineering, Czech Technical University in Prague 